\title{
Abstracts of the 7th International Conference on Lactoferrin / Résumés de la 7e Conférence internationale sur la lactoferrine
}

N. Kumagai, S. Tsunematsu, H. Hamano, K. Yamauchi, and K. Tsuchimoto

T. Kuhara, K. Yamauchi, and M. Takase

Zigang Tian, Jianhua Wang, Da Teng, Yalin Yang, Fan Zhang, Xingjun Feng, Lingyun Wei, Ying Fan, and Jin Luo

N.A. Thomson, A. Chand, and C.J. Fee

R. Sato, Y. Kobayashi, S. Kobayashi, J. Sato, Y. Yamada, Y. Naito, and J. Sasaki

J.G.M. Bolscher, M.I.A. Van der Kraan, K. Nazmi, H. Kalay, C. Grun, W. Van't Hof, E.C.I. Veerman, and A.V. Nieuw Amerongen

M. Kaito, K. Yamauchi, M. Iwasa, Y. Kobayashi, N. Fujita, H. Tanaka, S. Horike, M. Konishi, S. Watanabe, S. Teraguchi, and Y. Adachi

K. Mitsukawa, K. Otsuki, M. Sawada, K. Yakuwa, A. Hasegawa, Y. Sasaki, H. Chiba, M. Nagatsuka, and T. Okai

A.M. Degener, G. Longhi V, M. Mischitelli, C. Longhi, M.P. Conte, P. Valenti, L. Seganti, M. Marchetti, and F. Superti

H.E. Indyk and E.L. Filonzi

Zigang Tian, Jianhua Wang, Da Teng, Yalin Yang, Fan Zhang, Xingjun Feng, Lingyun Wei, Ying Fan, and Jin Luo

Rahman Md. Morshedur, Woan-Sub Kim, Toshiaki Ito, Haruto Kumura, and Keiichi Shimazaki

H. Wakabayashi, T. Andoh, K. Yamauchi, and M. Takase

Motoh Iwasa, Masahiko Kaito, Koji Yamauchi, Ryosuke Sugimoto, Mika Yamamoto, Naoki Nakagawa, Masayoshi Konishi, Shozo Watanabe, Susumu Teraguchi, and Yukihiko Adachi

K. Nakashima, Y. Yumoto, and Y. Furuichi
385

Oral administration of lactoferrin enhances viral decline in patients with genotype-1B-related chronic hepatitis $C$ treated with Peginterferon $\alpha-2 B$ plus ribavirin

385

Oral administration of lactoferrin raises NK cell activity in mice

386 Comparison of antimicrobial activity between lactoferricin B 15 derivative and antibiotics

Batch extraction of lactoferrin from raw whole milk

Modulatory effects of oral administration of entericcoated lactoferrin on neutrophil functions in Feline Immunodifiency Virus (FIV) infected cats

Novel antimicrobial Ifampin from lactoferrin released by a one-enzyme strategy

Efficacy of oral bovine lactoferrin on the interferon $\alpha$ plus ribavirin therapy for chronic hepatitis $C$ patients with genotype $1 \mathrm{~b}$ and a high viral load

388 Cervical inflammatory cytokines and other markers in cervical mucus of pregnant women with bacterial vaginosis

389 Lactoferrin inhibits human BK polyomavirus adsorption to Vero cells

Determination of lactoferrin in bovine milk, colostrum and infant formulas by optical biosenso analysis

390 Design and construction of tandem multimers lactoferricin B15 derivative for high-level expression in Escherichia coli

Visualization of lactoferrin binding to bifidobacteria

Antimicrobial activity of lactoferrin against Enterobacter Sakazakii

Lactoferrin inhibits lipid peroxidation in chronic hepatitis $\mathrm{C}$ patients

response of THP-1 cells 
S. Tomita, T. Uotani, N. Sueki, K. Fujimoto, J. Matsuyama, and Y. Benno

S.-A. Turner and N.A. Thomson

S. Kobayashi, R. Sato, O. Inanami, T. Yamamori, M. Kuwabara, Y. Naito, and M. Takase

K. Otsuki, K. Yakuwa, M. Sawada, K. Ichizuka, M. Nagatsuka, T. Kimura, and T. Okai

S. Farnaud, Z. Wang, R. Dybowski, E.W. Odell, and

R.W. Evans

Y. Yumoto, K. Nakashima, and Y. Furuichi

Jeremy H. Brock, Madalina Icriverzi, Mihaela Trif, Flori Chelu, Gabriela Negroiu, and Anca Roseanu

K. Kai, M. Itagaki, M. Uematsu, Y. Miyazaki, D. Fukumoto, I. Iwaski, H. Tsuchida, Y. Taguchi, S. Ishiguro, K. Kumagai, T. Yanaka, and M. Mochizuki

E.R. Sadchikova, I.L. Goldman, A.N. Krasnov, S.G. Kadulin, S.G. Georgieva, T.G. Ermolkevich, Ya. G. Gursky, N.V. Gnuchev, and G.P. Georgiev

Yokoyama Saichiro, Koshio Shunsuke, Teshima Shin-ichi, Ishikawa Manabu, ReyesCatacutan Mae, Takakura Natsuko, and Oshida Kyoichi

K. Kai, Y. Komine, K. Komine, M. Ohta, and K. Kumagai E.F. Haney, F. Lau, and H.J. Vogel

K. Higami, K. Ishii, M.L. Shinohara, T. Ikehara, H. Nagai, M. Watanabe, Y. Sumino, K. Miki, and K. Yamauchi

M.S. Nam, M.C. Cho, H.C. Bae, and D.Y. Yoon Tamaki Yukimichi and Ando Kunio Shaper Mirza, William H. Benjamin Jr., and David E. Briles

Shaper Mirza and David E. Briles

David E. Briles and Shaper Mirza
401
Promotional effect of bovine lactoferrin on the growth of bifidobacteria and their mechanism

Lactoferrin in bovine milk is not affected by strain of cow or level of feed

Bovine lactoferrin reduces concanavalin A-induced interferon- $\gamma$ expression of peripheral blood mononuclear cells in FIV-negative and FIV-positive cats

Effect of enteric-coated lactoferrin on constipation during pregnancy

Towards a tree-induction approach to antimicrobial activity analysis of cationic peptides

Distribution of human lactoferrin in periodontal pockets

Effect of lactoferrin on murine melanoma B16-F1 cells; possible mode of action

Efficacy and safety of lactoferrin as a new preventive reagent for bovine mastitis

Human lactoferrin in milk of transgenic animals

Effect of dietary bovine lactoferrin on tolerance to air exposure stress and response in branchial heatshock protein under low-salinity conditions in grouper Epinephelus coioides

Therapeutic effects of bovine lactoferrin on staphylococcal mastitis during non-lactating period

NMR structural studies of bovine and human lactoferrampin, novel antimicrobial peptides from lactoferrin

Long-term follow-up of chronic hepatitis $\mathrm{C}$ patients administered oral lactoferrin

Effects of lactoferrin on adipogenesis in 3t3-11 cells and obesity mice

Lactoferrin in dentistry

Killing of pneumococci by apolactoferrin is dependent on cleavage of apolactoferrin by the pneumococcal surface serine protease PrtA

Cleavage of human lactoferrin by a surfaceassociated serine protease (PrtA) of Streptococcus pneumoniae

PspA inhibits the antibacterial effect of lactoferrin on Streptococcus pneumoniae 
Jianhua Wang, X. Feng, A. Shan, D. Teng, Y. Yao, Y. Shao, S. Liu, and F. Zhang

K. Ishii, Y. Sumino, M. Kogame, K. Higami, M.I. Shinohara, K. Matsumaru, M.A. Shinohara, H. Nagai, M. Watanabe, K. Miki, K. Yamauchi, and S. Teraguchi
402 Fusion expression of bovine lactoferricin in Escherichia Coli

402 The efficacy of bovine lactoferrin and ifn- $\alpha 2 b$ combination therapy following 24-week regimen of ribavirin plus ifn- $\alpha 2$ b for chronic hepatits $C$ patients infected with genotype $1 \mathrm{~b}$ and high viral load 


\title{
ABSTRACTS / RÉSUMÉS
}

\section{Oral administration of lactoferrin enhances viral decline in patients with genotype-1B-related chronic hepatitis $C$ treated with Peginterferon $\alpha$ - 2B plus ribavirin}

\author{
N. Kumagai, S. Tsunematsu, H. Hamano, K. Yamauchi, and K. Tsuchimoto \\ Research Center for Liver Diseases, Kitasato Institute Hospital, Tokyo, Japan; Nutritional \\ Science Laboratory, Morinaga Milk Industry Co. Ltd, Zama, Japan
}

We previously reported that lactoferrin (Lf) lowers serum HCV-RNA levels in patients with genotype-1b-related chronic hepatitis $\mathrm{C}$ and that pre-administration of Lf improves the clinical outcome of treatment with interferon (IFN) plus ribavirin in patients with chronic hepatitis $\mathrm{C}$. Here, to evaluate the efficacy of Lf on viral kinetics, we studied HCV dynamics in patients with genotype $1 \mathrm{~b}$ and a high viral load who were randomly assigned to the following 2 different treatment groups: ( $i$ ) combination therapy involving Peginterferon $\alpha-2 b$ plus ribavirin (Peg-R); (ii) combination therapy (Peg-R) plus oral administration of Lf at a dose of $600 \mathrm{mg} /$ day (Peg-R-Lf).

Patient baseline characteristics between the Peg-R group $(n=11)$ and the Peg-R-Lf group $(n=9)$ were similar. Mean serum HCV-RNA levels at baseline in the Peg-R and Peg-R-Lf groups were $1500 \mathrm{KIU} / \mathrm{mL}$ and $1468 \mathrm{KIU} / \mathrm{mL}$, respectively. Undetectable HCV-RNA in serum at 12 weeks post-treatment was observed more frequently in the Peg-RLf group (4/6) than in the Peg-R group (3/10), although this difference was not statistically significant. Using a logtransformed linear regression model, the decline slope was calculated on serum HCV-RNA quantifications. As a result, early phase of viral decay (weeks 0-12) was significantly faster in the Peg-R-Lf group compared with those with Peg-R group.

In the Peginterferon plus ribavirin treatment, most patients with early viral response (EVR) defined at week 12 as an at least 2-log decline from baseline of the HCV-RNA level subsequently achieved a sustained viral response (SVR). Our results suggest that Lf enhanced antiviral effect in the early phase of HCV dynamics and helped EVR for the Peginterferon plus ribavirin treatment in patients with chronic hepatitis $\mathrm{C}$.

\section{Oral administration of lactoferrin raises NK cell activity in mice}

\author{
T. Kuhara, K. Yamauchi, and M. Takase \\ Nutritional Science laboratory, Morinaga Milk Industry Co. Ltd, Japan
}

There have been numerous studies indicating that lactoferrin (LF) influences various immune functions. Recently, some reports have shown that bovine LF (bLF) enhanced anti-microbial and anti-tumor immune activities when orally administered. We observed that oral bLF administration for 7 consecutive days augmented the population and number of natural killer (NK) cells in peripheral blood and spleen in a dose-dependent manner, and enhanced interferon- $\gamma$ production by NK cells. When poly(I:C) i.p. injection induced NK cell trafficking into the peritoneum, bLF oral administration increased NK cell accumulation. Oral administration of bLF immediately induced the elevation of portal interleukin-18 (IL-18) levels. In IL-18 knockout mice, bLF could not enhance the number of NK cells. However, NK cell cytotoxic activity and poly(I:C)-induced trafficking activity were enhanced by oral bLF administration even in IL-18 knockout mice. Furthermore, oral bLF administration augmented the expression of type-I interferons (interferon- $\alpha$ and $-\beta$ ) in 
Peyer's patch and the mesenteric lymph nodes. Oral administration of 2-chloroadenosine reduced the number of Peyer's patch cells, and remarkably prevented the ability of oral bLF administration for NK cell accumulation in the peritoneum after poly(I:C) i.p. injection. Collectively, these results indicated that orally administered bLF stimulated intestine-associated immune functions, including the production of IL-18 and type-I interferons, and then raised the NK cell activity.

\title{
Comparison of antimicrobial activity between lactoferricin B 15 derivative and antibiotics
}

\author{
Zigang Tian, Jianhua Wang, Da Teng, Yalin Yang, Fan Zhang, Xingjun Feng, \\ Lingyun Wei, Ying Fan, and Jin Luo \\ Gene Engineering Laboratory, Feed Research Institute, Chinese Academy of Agricultural \\ Sciences, Beijing 100081, China
}

The cationic antimicrobial peptide lactoferricin B15 (LfcinB15, FKCWRWQWRWKKLGA), was synthesized by chemical method. In vitro susceptibilities of LfcinB15 and four antibiotics for clinical useage (benzylpenicillin, chlortetracycline hydrochloride, streptomycin, doxycycline) were evaluated against several gram-positive and gram-negative bacteria and against Pichia pastoris GS115 using a modified microtitre broth dilution method by the Hancock laboratory. The tested peptide showed different antimicrobial activity against 6 species of microorganisms. Obviously, the grampositive bacteria are much more sensitive to LfcinB15 than the gram-negative bacteria are. LfcinB15 has more broadspectrum, but lower, antimicrobial activity against bacteria when compared with 4 kinds of traditional antibiotics. Its antimicrobial mechanism is unique and the characteristic of bacterial resistance forming has not been found. It can be expected that the methods and results of this study are helpful for the future evaluation and screening of other synthesized and recombinant LfcinB derivatives.

This project is funded by the Chinese National Hi-Tech Research and Development Programs Nos. 2004AA246040 and 2003AA241160-01.

\section{Batch extraction of lactoferrin from raw whole milk}

\author{
N.A. Thomson, A. Chand, and C.J. Fee \\ Dexcel Limited, Hamilton, New Zealand; Department of Materials and Process \\ Engineering, The University of Waikato, Hamilton, New Zealand
}

On-farm fractionation of milk proteins is a patented process that allows extraction of highly valuable components out of raw whole milk immediately after harvest. The production of high-value dairy proteins such as lactoferrin (LF) and lactoperoxidase (LP) normally involves extensive pretreatments of milk; for example, cooling for storage and transportation, centrifugation to remove fat, and precipitation or filtration to remove caseins. Preprocessing may cause loss in protein activity and yield or both. Similar processing is also applied for processing milk proteins such as recombinant lactoferrin (rLF) from transgenic animals.

LF and LP were used as model proteins to demonstrate the concept of direct capture of high-value components from milk. A cation exchanger, an SP Sepharose Fast Flow $^{\mathrm{TM}}$ (GE Healthcare, Uppsala, Sweden), was used in a single-stage stirred tank to extract LF. Raw whole milk from individual cows, representing a range of feed LF concentrations, was used for extractions. Experimental data obtained for resin-milk ratios of 0.010 to 0.024 over time were modelled using a composite non-linear model (CNL) (Rowe, G.E., et al. 1999. Biotechnol. Bioeng. 65(6)). The effects of adsorption rates, resin-milk ratios, feed LF concentrations, and adsorption capacity of resin on extraction were investigated. Maximum percentages of original LF extracted by SP Sepharose Fast Flow ${ }^{\mathrm{TM}}$ were 67\%, 80\%, 88\%, and $90 \%$ for extraction times of $5,10,20$, and $30 \mathrm{~min}$, respectively. Results from these batch experiments demonstrated that single-stage batch extractions can be used to extract LF. Resinmilk ratios and adsorption times can be manipulated to achieve the desired yields of LF. 


\title{
Modulatory effects of oral administration of enteric-coated lactoferrin on neutrophil functions in Feline Immunodifiency Virus (FIV) infected cats
}

\author{
R. Sato, Y. Kobayashi, S. Kobayashi, J. Sato, Y. Yamada, Y. Naito, and J. Sasaki \\ Department of Veterinary Internal Medicine, Iwate University, Japan
}

Feline immunodeficiency virus (FIV) infection shows a similar clinical time-course to human immunodeficiency virus (HIV) infection. We previously reported anti-inflammatory effects of bovine lactoferrin (bLF) on intractable stomatitis in cats infected with FIV, and we also clarified the regulatory effects of bLF on neutrophil functions in these cats. On the other hand, enteric-coated LF was made to avoid pepsin disintegration of the stomach. Recent studies reveal that enteric-coated LF has biological activites on both the central nerve system and lipid metabolism. In the present study, we investigated whether enteric-coated LF shows modulatory effects on neutrophil functions in the same way that bLF does in FIV-infected cats.

Healthy and FIV-infected cats were orally administered one enteric-coated LF tablet (containing $90 \mathrm{mg}$ of bLF; 18$28 \mathrm{mg} / \mathrm{kg}$ body mass) for $7 \mathrm{~d}$ and neutrophil functions were investigated before and $7 \mathrm{~d}$ post-administration initiation. Bovine LF was also administered for $7 \mathrm{~d}$ (therapeutic dose;
$40 \mathrm{mg} / \mathrm{kg}$ body mass) and neutrophil functions were investigated in the same manner.

Activities of neutrophil phagocytosis were significantly increased after administration of enteric-coated LF and bLF in FIV-infected cats. Luminol-induced chemiluminescence of neutrophils stimulated by opsonized zymozan did not show significant changes in either cat group. Neutrophil adherence activity was decreased after administration of enteric-coated LF and bLF. Enteric-coated LF showed modulating effects on neutrophil functions in FIV-infected similar to the effects of bLF. Therefore, it seemed that enteric-coated tablets enable the reduction of the dose of bLF. Bovine LF induces anti-inflammatory effects in FIV-infected cats with intractable stomatitis through its neutrophil-regulation activities. Therefore, there is the possibility that a smaller than therapeutic dose of enteric-coated LF may show anti-inflammatory effects in FIV-infected cats.

\section{Novel antimicrobial Ifampin from lactoferrin released by a one-enzyme strategy}

\author{
J.G.M. Bolscher, M.I.A. Van der Kraan, K. Nazmi, H. Kalay, C. Grun, W. Van't Hof, \\ E.C.I. Veerman, and A.V. Nieuw Amerongen \\ Department of Oral Biochemistry, ACTA, Amsterdam, and Department of Molecular Cell \\ Biology and Immunology, VU Medisch Centrum, Amsterdam, Netherlands
}

Antimicrobial peptides have been found throughout nature, yet antimicrobial domains may still lie hidden within a wide variety of multifunctional proteins. The objective of this study was to develop a rational strategy to select domains of interest based on the presumed common features of antimicrobial peptides, and to functionally release these from accessible and safe proteins. To demonstrate the proof of principle, bovine lactoferrin (bLF) was screened for suitable stretches, including a cationic amphipathic $\alpha$-helix. Subsequently, proteolytic enzymes were selected that can theoretically release the potential antimicrobial sequences. These sequences were chemically synthesized or isolated from the native molecule and subsequently tested for their killing activity. Peptides corresponding to the identified antimicrobial domain, designated LFampins, indeed possessed broad-spectrum antimicrobial activity. In silico proteolysis simulations of bLF with selected endoproteinases predicted the liberation of peptides containing the required LFampin sequence. Three predicted peptides were synthesized and tested for their biological activity, elucidating that a single enzyme (endoproteinase AspN) was sufficient to obtain an antimicrobial peptide. The final proof of principle demonstrated that a 32-mer fragment, LFampin 265-296, isolated from the AspN-digestion of bLF, possessed antimicrobial activity as strong as that of the synthetic LFampin. Moreover, AspN digestion of bLF increased its antimicrobial activity by release of antimicrobial stretches.

Financially supported by the Dutch Technology Foundation, STW, grant No. VTH5180; the Dutch Dairy Association; and DMV International. 


\title{
Efficacy of oral bovine lactoferrin on the interferon $\alpha$ plus ribavirin therapy for chronic hepatitis $C$ patients with genotype $1 \mathrm{~b}$ and a high viral load
}

\author{
M. Kaito, K. Yamauchi, M. Iwasa, Y. Kobayashi, N. Fujita, H. Tanaka, S. Horike, \\ M. Konishi, S. Watanabe, S. Teraguchi, and Y. Adachi \\ Department of Gastroenterology and Hepatology, Division of Clinical Medicine and \\ Biomedical Sciences, Institute of Medical Science, Mie University, 1.2-174 Edobashi, Tsu, \\ Mie 514-8507, Japan
}

Many types of interferon (IFN) therapy for chronic hepatitis $\mathrm{C}$ (CHC) have been developed, but the efficacy of these therapies has been not high enough. Therefore, various attempts to enhance the efficacy of IFN have been tried.

In the present study, we examined the efficacy of LF at a high dose $(3.6 \mathrm{~g} / \mathrm{d})$ for 8 weeks before IFN-ribavirin (IFN-RBV) therapy. Eighty-seven $\mathrm{CHC}$ patients with genotype $1 \mathrm{~b}$ and a high viral load (the mean load was $>2500 \times 10^{4}$ copies $/ \mathrm{mL}$ ) were randomized to an LF group and a control group. Patients in both groups received the IFN-RBV therapy for 24 weeks. From 8 weeks before the start until the end of INF-RBV therapy, patients in the LF group received bovine LF (Morinaga Milk Industry) orally at a dose of $3.6 \mathrm{~g} / \mathrm{d}$.
There was no significant difference in the sustained virological response (SVR) between the 2 groups. Although INF-RBV therapy achieved a similar SVR in the 2 groups, the viral load was reduced significantly in the LF group. The SVR of the LF subgroup, with a decrease in viral load by at least $50 \%$ owing to LF administration, was significantly higher than in the control group.

It is not sufficient to cure CHC patients even with the most recent therapy (PEG-IFN plus RBV therapy), the SVR of which is only $47.6 \%$. Addition of LF treatment to IFN therapy is expected to increase the chance of recovery in patients who respond to $\mathrm{LF}$.

\section{Cervical inflammatory cytokines and other markers in cervical mucus of pregnant women with bacterial vaginosis}

\author{
K. Mitsukawa, K. Otsuki, M. Sawada, K. Yakuwa, A. Hasegawa, Y. Sasaki, H. Chiba, \\ M. Nagatsuka, and T. Okai \\ Department of Obstetrics and Gynecology, Showa University School of Medicine, Tokyo, \\ Japan
}

There is little debate that chorioamnionitis (CAM) results in preterm delivery from an ascending infection caused by bacterial vaginosis and cervicitis, both thought to occur before CAM. Many other studies have already demonstrated that lower genital-tract inflammation and infection are risk factors for preterm delivery. In this study, we determined concentrations of interleukin-6 (IL-6), interleukin-8 (IL-8), lactoferrin (LF), and $\alpha$-defencine $(\alpha-\mathrm{DF})$ in cervical mucus of pregnant women and analyzed their relation to bacterial vaginosis.

After obtaining both approval from the ethics committee and patient consent, we obtained cervical mucus samples from 87 pregnant women: 67 with uneventful pregnancies (group A), 9 with intermediate type (group B), and 13 who had bacterial vaginosis (group $\mathrm{C}$ ). The samples were analyzed for LF, DF, IL-6, IL-8, and elastase (EL).

The LF, IL-6, and EL concentrations in group C were significantly higher $(p<0.05)$ than in group $\mathrm{A}$. The $\alpha$-DF and IL-8 concentrations in group $\mathrm{C}$ were higher than in group $\mathrm{A}$. There were no significant changes in concentrations of LF, $\alpha$-DF, IL-6, IL-8, and EL in group B compared with group A. 
The higher LF, IL-6, and EL concentrations in cases of bacterial vaginosis may indicate faster defense in the cervix. Consequently, measurement of these inflammatory marker concentrations may lead to early detection of lower genitaltract inflammation and infection.

\title{
Lactoferrin inhibits human BK polyomavirus adsorption to Vero cells
}

\author{
A.M. Degener, G. Longhi V, M. Mischitelli, C. Longhi, M.P. Conte, P. Valenti, \\ L. Seganti, M. Marchetti, and F. Superti \\ Departments of Experimental Medicine and Pathology, and Public Health, La Sapienza \\ University, Rome, Italy; Department of Experimental Medicine, II University of Naples, \\ Naples, Italy; Department of Technology and Health, National Institute of Health, Rome, \\ Italy
}

$\mathrm{BK}$ virus (BKV) is a human naked polyomavirus responsible for productive, persistent, and latent infections of the urinary tract. The presence of its DNA has been revealed in various tumors. The severity of BKV infection in immunocompromised hosts has improved the search for selective antiviral agents, but hitherto only few effective compounds have been identified. Lactoferrin (Lf), a member of the transferrin family, is a biglobular iron-binding glycoprotein found in milk, exocrine secretions of mammals, and in secondary granules of polymorphonuclear neutrophiles. Lf was established as playing an important role in the defence against various pathogenic microorganisms. Previously, we demonstrated that $\mathrm{Lf}$ is a potent inhibitor of infection by some enveloped and naked viruses in different cell systems. In this research, we investigated the effect of this glycoprotein on BKV infection. The experiments were carried out synchronizing infection by a temperature shift and viral replication in Vero cells and was monitored measuring BKV DNA synthesis by semiquantitative PCR assay of the TAG region. The results showed that inhibition of $\mathrm{BKV}$ replication already occurred when Lf was added during viral adsorption. Moreover, pre-incubation of Lf with BKV before infection inhibited viral replication by $98 \%$, suggesting a specific binding of Lf to the virions. Finally, the direct interaction of Lf to BKV particles was demonstrated by hemagglutination inhibition assays and visualized by transmission electron microscopy. These results demonstrated that, similarly to data obtained with other viruses, Lf inhibits the early steps of infection through a direct interaction with viral structures. Further studies will be carried out to better clarify the effects of Lf on human polyomavirus replication.

\section{Determination of lactoferrin in bovine milk, colostrum and infant formulas by optical biosenso analysis}

\author{
H.E. Indyk and E.L. Filonzi \\ Fonterra, PO Box 7, Waitoa, Waikato, New Zealand
}

An automated, rapid, sensitive, and label-free biosensorbased immunoassay for lactoferrin in bovine milk using surface plasmon resonance (SPR) optical detection is described. Lactoferrin content was estimated from its specific interaction with an anti-bovine lactoferrin antibody immobilized on the sensor surface in a direct binding assay format. Samples were prepared for analysis by direct dilution into buffer. Analysis conditions, including ligand immobilization, flowrate, contact time, and regeneration, were defined and nonspecific binding considerations evaluated. Performance pa- rameters included a working range of $0-1000 \mathrm{ng} / \mathrm{mL}$, a method-detection limit of $19.9 \mathrm{~g} / \mathrm{mL}$ in fluid milk, overall instrument response $\mathrm{RSD}_{\mathrm{R}}$ of $3.50 \%$, a mean inter-assay $\mathrm{RSD}_{\mathrm{R}}$ of $10.8 \%$ for milk powder, and surface stability over approximately 500 samples. The technique was applied to the measurement of lactoferrin content of consumer milks, colostrum, and infant formulas. The influence on lactoferrin content of temporal change during early bovine lactation, and the effects of heat on denaturation, were evaluated. 


\title{
Design and construction of tandem multimers lactoferricin B15 derivative for high-level expression in Escherichia coli
}

\author{
Zigang Tian, Jianhua Wang, Da Teng, Yalin Yang, Fan Zhang, Xingjun Feng, \\ Lingyun Wei, Ying Fan, and Jin Luo \\ Gene Engineering Laboratory, Feed Research Institute, Chinese Academy of Agricultural \\ Sciences, Beijing 100081, China
}

A combination of multimerization and a Trx fusion protein were used to mask the toxicity of a lactoferricin B15 (LfcinB15) derivative in Escherichia coli BL21 (DE3). The $\mathrm{CNBr}$ recognition site was introduced to the $\mathrm{N}$ - and $\mathrm{C}$-terminals of the peptide to facilitate the final release and separation of the recombinant peptide. The synthetic genes were ligated tandemly by cohesive ends to form multimerization genes, then ligated with linearized and dephosphorylated pET32a(+) by BamHI and Hind adapters. Transformation and screening was then carried out via E. coli DH5 $\alpha$ and BL21(DE3) competent cells, respectively. The recombinant plasmids were confirmed by PCR analysis and DNA se- quencing. Finally, 8 recombinant plasmids, pET32LfcinB $15-1,-2,-3,-4,-5,-6,-7$, and -9 , were obtained in which difference amongst recombinants, even if it occurred in only one copy, was clearly visible. DNA sequencing results showed that all 8 recombinants were in frame and their sequences were also correct. This is an important basis for further research on expression, purification, and characterization of the recombinant peptide.

This project is funded by the Chinese National Hi-Tech Research and Development Programs Nos. 2004AA246040 and 2003AA241160-01.

\section{Visualization of lactoferrin binding to bifidobacteria}

\author{
Rahman Md. Morshedur, Woan-Sub Kim, Toshiaki Ito, Haruto Kumura, and Kei- \\ ichi Shimazaki \\ Laboratory of Dairy Science, Hokkaido University, Japan
}

The antimicrobial activity of lactoferrin has been well established for many years. However, lactoferrin does not inhibit the growth of certain lactic bacteria, including bifidobacteria; rather, it stimulates their growth. Several studies have reported that human or bovine lactoferrin promotes the growth of bifidobacteria in vitro and in vivo.

Currently, the most important issue is how lactoferrin promotes the growth of bifidobacteria. It is reported that many microorganisms have lactoferrin-binding proteins or receptors. Therefore, one possible mechanism of stimulating the growth of bifidobacteria is the presence of lactoferrin-binding protein in bifidobacteria. Our previous studies revealed the presence of such a binding protein.

In this study, we visualized the binding of bovine lacto- ferrin to bifidobacterial cells (B. infantis 21143 I 63-7) under native conditions. Binding of lactoferrin was inspected by confocal laser scanning microscopy (CLSM) using biotinylated lactoferrin and FITC-conjugated streptavidin and also by transmission electron microscopy (TEM) using biotinylated lactoferrin and gold-conjugated streptavidin. Lactoferrin-binding protein in the membrane fraction of bifidobacterial cells was also investigated by far-Western blotting using biotinylated lactoferrin and horseradish peroxidase conjugated streptavidin.

The results show the binding pattern of bovine lactoferrin to bifidobacterial cells under native conditions and also supports the previous findings by far-Western blotting showing the presence of lactoferrin-binding protein in bifidobacteria. 


\title{
Antimicrobial activity of lactoferrin against Enterobacter Sakazakii
}

\author{
H. Wakabayashi, T. Andoh, K. Yamauchi, and M. Takase \\ Nutritional Science Laboratory, Morinaga Milk Industry Co. Ltd, Japan
}

Enterobacter sakazakii is an opportunistic pathogen implicated in outbreaks of meningitis or enteritis with high mortality, especially in neonates and infants. The effect of host defence factors on the bacterium has rarely been reported. We investigated whether lactoferrin (LF) or its derivatives is effective for inhibition of the bacterium. Minimum inhibitory concentration (MIC) testing was performed against Escherichia coli $\mathrm{O} 111$ and E. sakazakii ATCC51329 by $17 \mathrm{~h}$ incubation in $1 \%$ peptone broth with approximately $10^{5}$ cells $/ \mathrm{mL}$ of inoculum. MIC $(\mu \mathrm{g} / \mathrm{mL})$ against these strains was 2000 and 250 for bovine LF, >8000 and 250 for human LF, 250 and 500 for bovine LF-pepsin hydrolysate, and 16 and 31 for lactoferricin (LFcin) B, respectively. Bovine LF at $4000 \mu \mathrm{g} / \mathrm{mL}$ exhibited a killing effect on E. sakazakii. These results suggest that E. sakazakii may be more sensitive to the bacteriostatic effect of LF through iron deprivation than to the bactericidal effect mediated by the LFcin domain or peptide. Now, we are extending the investigation by increasing a number of test strains.

\section{Lactoferrin inhibits lipid peroxidation in chronic hepatitis C patients}

\author{
Motoh Iwasa, Masahiko Kaito, Koji Yamauchi, Ryosuke Sugimoto, Mika Yamamoto, \\ Naoki Nakagawa, Masayoshi Konishi, Shozo Watanabe, Susumu Teraguchi, and \\ Yukihiko Adachi \\ Department of Gastroenterology and Hepatology, Division of Clinical Medicine and \\ Biomedical Sciences, Institute of Medical Science, Mie University, 1.2-174 Edobashi, Tsu, \\ Mie 514-8507, Japan
}

Oxidative stress plays an important role in the pathologenesis of chronic hepatitis $\mathrm{C}$ (CHC). Trials with antioxidants have been carried out to influence the progress of the disease. Bovine lactoferrin (bLF), a milk protein of the irontransporter family, is commercially available as a health food in Japan. Previous studies have shown that LF inhibits lipid peroxidation in vitro. The present study evaluated the effects of bLF on plasma 8-isoprostane, serum aminotransferase levels, viral load, and iron metabolism markers in patients with $\mathrm{CHC}$.

Fifty-eight outpatients with $\mathrm{CHC}$ were enrolled in the present study. The inclusion criteria were as follows: high serum levels of $\mathrm{HCV}$ RNA (more than $100 \mathrm{kIU} / \mathrm{mL}$ as measured by amplicor monitor assay and serum HCV genotype $1 \mathrm{~b}$ ), and absence of other causes of $\mathrm{CH}$. They were randomly assigned into 2 groups. Thirty-seven patients (23 men and 14 women; mean age 56 years) received oral bLF for 2 months at the daily dose of $3.6 \mathrm{~g}$ (Nutritional Science Laboratory, Morinaga Milk Industry, Zama, Japan). Twenty-one patients (13 men and 8 women; mean age 58 years) that were not treated with bLF served as controls.
During the observation periods, other medications were not used.

At entry, the plasma 8-isoprostane was significantly elevated in $\mathrm{CHC}$ patients as compared with controls. There was no significant difference between the 2 groups regarding age, sex, serum aminotransferase, HCV RNA levels, or iron metabolism markers. Plasma 8-isoprostane levels significantly $(p<0.05)$ decreased from $8.9 \pm 3.9$ to $7.6 \pm 4.6 \mathrm{pg} /$ $\mathrm{mL}$ after bLF treatment. In the bLF group, AST and ALT levels significantly decreased from $62 \pm 52$ to $46 \pm 34$ IU/L and $84 \pm 78$ to $61 \pm 54 \mathrm{IU} / \mathrm{L}$, respectively $(p<0.05)$. The serum levels of viral load, iron, ferritin, and transferring saturation remained unchanged throughout the study in both groups.

Increased oxidative stress, as measured by plasma level of 8-isoprostane, was observed in patients with CHC. Lipid peroxidation induced liver damage was reduced by bLF in patients with viral hepatitis $\mathrm{C}$. The results of this study suggest that bLF can be used as a supportive therapy to combat liver damage caused by lipid peroxidation. 


\title{
Effects of human lactoferrin on the chemotactic response of THP-1 cells
}

\author{
K. Nakashima, Y. Yumoto, and Y. Furuichi \\ Hokkaido University of Health Sciences, Department of of Periodontology, School of \\ Dentistry, 1757 Kanazawa, Tobetsu-cho, Ishikari-gun, Hokkaido 061-0293, Japan
}

Gingival crevicular fluid in periodontal pockets contains LPS derived from periodontopathic bacteria and human lactoferrin (hLf) released by neutrophils. HLf inhibits LPS interaction with CD14, decreasing TNF- $\alpha$ release from LPSstimulated monocytic cells. Bacterial components, e.g., fMLP and LPS, also function as chemoattractants. However, whether hLf affects the chemotactic response of monocytic cells is unclear. This study examined the effects of hLf on chemotactic response to various chemoattractants. Human monocytic cells, THP-1 cells, were cultured in RPMI 1640 medium in the presence of $1 \alpha, 25$-dihydroxyvitamin $\mathrm{D}_{3}$ for 24 h. THP-1 cells were then washed and transferred to the upper chamber set in a 24-well culture plate; the lower chamber contained either fMLP, LPS from Escherichia coli, IL-8, MCP-1, or hLf (Boyden chamber method). Following a $1 \mathrm{~h}$ incubation, the membrane on the floor of the upper chamber was removed and stained with Diff-Quik ${ }^{\circledR}$ to meas- ure absorbance at $595 \mathrm{~nm}$. Quantification of the chemotactic response of cells was evaluated via calculation of the chemotactic index (CI) (absorbance in the presence of chemoattractant vs. absorbance in the absence of chemoattractant). CIs for fMLP, LPS, IL-8, and MCP-1 were 2.00, 2.75, 1.46, and 1.59 , respectively. CIs changed to $1.77,1.90,3.27$, and 3.32, respectively, after additions of hLf. CI for hLf was 1.50; furthermore, it did not change significantly after introduction of polymyxin $\mathrm{B}$, which indicated that the chemotactic response is not due to contamination of LPS. IL-8 and MCP-1 levels were significantly higher in the upper chamber in comparison with the lower chamber upon addition to the lower chamber of hLf as a chemoattractant. These findings suggest that hLf, which acts as a chemoattractant while suppressing enhanced chemotactic response to LPS, plays an important role in regulation of monocytic cell chemotaxis.

\section{Promotional effect of bovine lactoferrin on the growth of bifidobacteria and their mechanism}

\author{
S. Tomita, T. Uotani, N. Sueki, K. Fujimoto, J. Matsuyama, and Y. Benno \\ Faculty of Agriculture, Tamagawa University; Japan Collection of Microorganisms, RIKEN \\ BioResource Center
}

Lactoferrin (LF) has a bacteriostatic effect against putrefactive bacteria such as clostridia, but does not inhibit the growth of beneficial bacteria such as bifidobacteria. Bifidobacteria are probiotics that have been implicated in a number of beneficial effects such as prevention of gastrointestinal infections in humans and animals. The aim of this study is to clarify the mechanism for the growth promotional effect of LF on bifidobacteria.

Bifidobacteria strains in GAM liquid medium with LF were incubated anaerobically at $37{ }^{\circ} \mathrm{C}$ for $8 \mathrm{~h}$. Viable counts were determined by plating serial dilutions onto GAM agar plates. The growth of Bifidobacterium breve JCM1192 with LF increased about $20 \%$ compared with that without LF, but $B$. breve JCM7017 was barely affected. The binding abilites of LF to the cells of B. breve JCM1192 and JCM7017 were observed by an enzyme-linked ligand-binding assay using a horseradish peroxidase - LF (HRPO-LF) conjugate. The binding ability of LF to the cells of $B$. breve JCM1192 was significantly higher than that to cells of JCM7017. The extents of the binding of apo-LF to the cells of each strain were the same as those of Fe-LF. The LF binding site on the bifidobacterial cell was investigated. Detection of an LF-binding protein (BP) in surface components of cells extracted with guanidine hydrochloride was done by 2-dimensional PAGE andWestern blotting using HRPO-LF conjugate. Two spots corresponding to LF-BP were detected in $B$. breve JCM1192, and had relative molecular masses of about $36 \mathrm{kDa}$ and isoelectric points of about 6.3 and 6.4 , respectively. However, BP was not detected in $B$. breve JCM7017. BP binding to other milk proteins was not confirmed in this study.

These results suggest that the growth promotional effect of LF on bifidobacteria may be mainly responsible for LF binding to LF-BP on the cell surface. 


\title{
Lactoferrin in bovine milk is not affected by strain of cow or level of feed
}

\author{
S.-A. Turner and N.A. Thomson \\ Dexcel Ltd, Cnr. Ruakura and Morrinsville Roads, Private Bag 3221, Hamilton, \\ New Zealand
}

Methods of increasing the concentration of lactoferrin (Lf) in bovine milk could be valuable to processors interested in extracting Lf from milk. Previous work has shown that time of year, stage of lactation, and strain of cow affect milk Lf concentration. To investigate whether Lf in milk could be elevated by the selection of cows of particular strains, and (or) feeding a particular level of feed, a study was undertaken utilizing the milk samples collected from 6 herds of cows being farmed as part of a larger study to evaluate the performance of 3 strains of Holstein-Friesian cows in a range of farming systems. The trial included two highbreeding-worth (\$BW) strains of either North American (OS90) or New Zealand (NZ90) genetics, and one low \$BW strain of NZ genetics (NZ70) in 6 farm systems designed to provide feed allowances ranging from 5.0 to $6.5 \mathrm{tDM} / \mathrm{cow} \bullet \mathrm{y}^{-1}$. Cows were grazed on a pasture, with the 6 and 6.5 tDM herds being offered maize silage in the paddock during pasture deficit periods. Herds examined were NZ70s at 5.5 tDM; NZ90s at 5, 5.5, 6, and 6.5 tDM; and OS90s at 5.5 tDM and contained 15 (NZ70s) or 20 (NZ90 and OS90) cows/herd. Milk samples were collected individually from all cows in September (47 d in milk; DIM), November (96 DIM), and February (197 DIM) in both the 2002-2003 and 2003-2004 seasons. Data are the average of both seasons. No effect of strain on Lf concentrations was apparent for cows in the 5.5 tDM herds in September (65, 99, 65 mg/L; NZ70, NZ90, OS90, respectively), November (73, 99, $112 \mathrm{mg} / \mathrm{L})$, or February $(90,109,99 \mathrm{mg} / \mathrm{L})$. No effect of feed level on Lf concentrations was apparent for the NZ90 cows in September (93, 99, 120, $99 \mathrm{mg} / \mathrm{L} ; 5,5.5,6$, 6.5 tDM, respectively), November (111, 93, 136, $124 \mathrm{mg} /$ $\mathrm{L})$, or February $(136,109,136,136 \mathrm{mg} / \mathrm{L})$. The lack of strain effect on Lf concentrations is contradictory to previously published results and may suggest that the OS90 cows were not receiving adequate nutrition at the comparatively low feeding level of $5.5 \mathrm{tDM} / \mathrm{cow} \bullet \mathrm{y}^{-1}$. These results also suggest that feeding level cannot be used to manipulate Lf concentrations in the milk of NZ90 pasture-fed Friesian cows.

\section{Bovine lactoferrin reduces concanavalin A- induced interferon- $\gamma$ expression of peripheral blood mononuclear cells in FIV-negative and FIV- positive cats}

\author{
S. Kobayashi, R. Sato, O. Inanami, T. Yamamori, M. Kuwabara, Y. Naito, and \\ M. Takase \\ Iwate University, Department of Veterinary Internal Medicine, Faculty of Agriculture, 3-18- \\ 8, Ueda, Morioka 020-8550, Japan
}

We previously reported anti-inflammatory effects of bovine LF (bLF) on intractable stomatitis in cats infected with feline immunodeficiency virus (FIV), a model of HIV infection. Futhermore, it revealed that bLF modulates neutrophil functions including phagocytosis and adherence. In this study, to clarify the anti-inflammatory effects of bLF, we investigated the effect of bLF on feline lymphocyte functions. In addition, we also examined the relation between the effect of bLF and the intracellular-activated protein kinases.
Peripheral blood mononuclear cells (PBMCs) were obtained from FIV-negative and FIV-positive cats. The proliferation was measured using the MTT colorimetric method. Bovine LF was added to the PBMCs $30 \mathrm{~min}$ before and 40 min after concanavalin A (ConA) stimulation. After RNA extraction, interferon- $\gamma$, interleukin- $1 \beta$, TNF- $\alpha$, and interleukin-12 p40 mRNA expression were detected by semiquantitative or real-time RT-PCR. The activation of extracellular signal regulated kinases (ERKs) and protein ty- 
rosine kinases (PTKs) of PBMCs was examined by Western blotting, which used the phosphorylated antibody in FIVnegative cats.

LF inhibited the ConA-induced PBMC proliferation in a dose-dependent manner. RT-PCR showed a ConA-induced increase of interferon- $\gamma$ mRNA expression, but not of interleukin-1 $\beta$, TNF- $\alpha$, or interleukin-12 p40 mRNA in both feline PBMCs. This ConA-induced increase of interferon- $\gamma$ mRNA expression was significantly inhibited by bLF addition not only $30 \mathrm{~min}$ before but also $40 \mathrm{~min}$ after ConA stimulation. Especially, the inhibitory effect was more strong in FIV-positive cats with intractable stomatitis. Using specific kinase inhibitors of ERK (PD098059) and PTK (genistein), we found that the increase of ConA-induced interferon- $\gamma$ mRNA expression was ERK and PTK dependent. Western blotting revealed that the activation of PTK and ERK kinases had almost disappeared by $40 \mathrm{~min}$ after ConA stimulation.

These results suggest that bLF inhibits the ConA-induced interferon- $\gamma$ mRNA expression by abrogation of intracellular inflammatory effects of bLF in vivo, which seems to be mediated in part through lymphocyte functions such as local cytokine production.

\title{
Effect of enteric-coated lactoferrin on constipation during pregnancy
}

\author{
K. Otsuki, K. Yakuwa, M. Sawada, K. Ichizuka, M. Nagatsuka, T. Kimura, and \\ T. Okai \\ Department of Obstetrics and Gynecology, Showa University School of Medicine, Tokyo \\ 142-8666, Japan
}

Symptoms of constipation, menstrual colic, discharge, and preterm labor are related to menstruation, aging, lower genital-tract infections, and pregnancy. We evaluated the alleviation of these symptoms, particularly during pregnancy, by oral administration of enteric-coated lactoferrin (LF, tablet, NRL-Pharma Inc.).

This study was approved by the Showa University School of Medicine ethics committee. Following an explanation of this study, each of the 7 women (6 at 19 to 32 weeks of pregnancy at the start of the study and 1 non-pregnant subject) agreed to participate. All 7 were taking medication for constipation (typically $1000-2000 \mathrm{mg} / \mathrm{d} \mathrm{MgO}_{2}$ ). Three of the 6 pregnant women were hospitalized for observation owing to preterm labor and cervical insufficiency, while the remaining 3 showed normal course and outcome and were not taking any additional known anti-inflammatory medications. Before and after the administration of LF $(400 \mathrm{mg} / \mathrm{d}$ for at least 1 month), symptoms of constipation, menstrual colic, discharge, and preterm labor were observed and recorded.
Cervical mucus and vaginal fluid samples were collected for the determination of elastase concentration and for bacterial culturing, respectively.

Six of the 7 subjects experienced remarkable reductions in both the dose and frequency usage of constipation medicine within a few days of starting LF administration. Further, symptoms of constipation eased and frequency of bowel movements increased. Bacterial flora of the vaginal fluid and inflammatory markers in cervical mucus did not change significantly.

As previously reported, LF administration has the potential to reduce many minor discomforts associated with pregnancy, particularly common constipation. While the mechanisms remain unclear, direct and indirect (combination with bile) effects were suspected to affect the bacterial flora of the digestive ducts (the small intestine and the rectum) in the mitigation of constipation. Further studies on clinical use of LF, particularly for pregnant women, are needed.

\section{Towards a tree-induction approach to antimicrobial activity analysis of cationic peptides}

\author{
S. Farnaud, Z. Wang, R. Dybowski, E.W. Odell, and R.W. Evans \\ King's College London, Randall Division of Cell and Molecular Biophysics, Guy's \\ Campus, New Hunt House 3/6b, London SE1 1UL, UK
}

Owing to their ever-increasing number and diversity, antimicrobial cationic peptides can be a valuable tool against the growing occurrence of resistant microorganisms. Their modes of action, however, remain unidentified. The antimicrobial properties of lactoferrins are partly attributed to the lactoferricins, the antimicrobial peptides that correspond to 
the N-terminus region of lactoferrin after pepsin cleavage of human and bovine lactoferrin. To define the relationship between the structure of these peptides and their antimicrobial activity, quantitative structure-activity relationship (QSAR) studies have been performed using a tree-induction approach and are presented to illustrate their ease of interpretation and potential applicability in structure-function studies of cationic antimicrobial peptides.

\title{
Distribution of human lactoferrin in periodontal pockets
}

\author{
Y. Yumoto, K. Nakashima, and Y. Furuichi \\ Department of Periodontology, School of Dentistry, Health Sciences University of \\ Hokkaido, 1757 Kanazawa, Tubetsu-cho, Ishikari-gun, Hokkaido 061-0293, Japan
}

Periodontopathic bacteria endotoxin permeates dental root surfaces exposed to periodontal pockets, eliciting an inflammatory response. Human lactoferrin (hLf) inhibits interaction between LPS and CD14; thus, hLf released by neutrophils may participate in the inflammatory response. The present study examined the distribution of hLf in periodontal pockets and its roles in the pathogenesis of periodontitis. Nineteen teeth not salvageable by periodontal treatment were extracted with informed consent of the patients. Five extracted teeth were incubated with anti-hLf antibody and Alexa 532-conjugated secondary antibody; subsequently, fluorescence was measured. Powdered samples were prepared from 14 extracted roots. Samples were partitioned into 3 groups based on location: layers I, II, and III (from the root surface to the pulp cavity). Samples were then washed with PBS; hLf and endotoxin levels in the supernatant were measured. Additionally, hLf levels in gingival crevicular fluid (GCF), saliva, and supra- and sub-gingival dental plaque of healthy volunteers were determined. Furthermore, human monocytic cells, THP-1 cells, were cultured with hLf and LPS from Escherichia coli for $24 \mathrm{~h}$, after which LDH activity and cytokine levels (TNF-a, IL-8 and MCP-1) in culture supernatants were measured. Areas displaying fluorescence were consistent with those areas characterized by calculus. Significantly higher hLf and endotoxin levels were detected in layer I from areas with calculus relative to layer I from areas lacking calculus. GCF demonstrated higher hLf levels in comparison with saliva. LDH activity and cytokine levels increased following LPS stimulation; however, LDH activity and TNF- $\alpha$ levels decreased upon introduction of hLf. These findings suggest that hLf released by neutrophils is bound to LPS in periodontal pockets, which leads to inhibition of $\mathrm{TNF}-\alpha$ release and enhancement of chemokine release from monocytic cells.

\section{Effect of lactoferrin on murine melanoma B16-F1 cells; possible mode of action}

\author{
Jeremy H. Brock, Madalina Icriverzi, Mihaela Trif, Flori Chelu, Gabriela Negroiu, \\ and Anca Roseanu \\ Department of Immunology, Western Infirmary, Glasgow G11 6NT, Scotland, UK; Institute \\ of Biochemistry, Splaiul Independentei 296, 060031 Bucharest, Romania
}

Lactoferrin (Lf) is a key multifunctional iron-binding glycoprotein found throughout the body. Many different roles have been attributed to lactoferrin, including the primary defence against tumorigenesis. Several studies have reported that Lf supresses the growth of tumour cells in vitro and reduces experimental metastasis induced in mice. However the mechanism of Lf action is still under debate.

We previously reported that $\mathrm{Lf}$ is cytotoxic to murine melanoma B16-F1 cells by inducing a reduction of cell viability and morphological modifications probably determined by an apoptotic process.
The aim of this study was to investigate the interaction of Lf with murine melanoma B16-F1 cells and its effect at the intracellular level.

We found that Lf specifically binds to the cell surface and is internalized into B16-F1 cells using immunofluorescence and an ELISA-type method. We also analyzed the effect of Lf on the expression of tyrosinases, TRP-1 and TRP-2. These proteins belong to the tyrosinase-related proteins (TRP) family and are enzymes catalyzing the major steps in the synthesis of melanins, the pigment produced by melanocytes. TRP-1 and TRP-2 are also involved in other cellular 
functions, such as cell viability, growth, and morphology of melanocytes. It has also been suggested that TRP-2 might play a role in modulating apoptotic pathways by controlling the concentration of 5,6-dihydroxyindole, a very toxic intermediate that, in the absence of the enzyme, would accumulate in melanocytes. Our experiments performed by SDSPAGE and Western blot with specific antibodies revealed that Lf down regulated the expression of TRP-2. No effect on tyrosinase and TRP-1 expression in B16-F1 cells was observed.

These data suggest that $\mathrm{Lf}$ is cytotoxic to B16-F1 cells by down-regulating TRP-2 expression and thus affecting its capacity to modulate apoptotic pathways.

\title{
Efficacy and safety of lactoferrin as a new preventive reagent for bovine mastitis
}

\author{
K. Kai, M. Itagaki, M. Uematsu, Y. Miyazaki, D. Fukumoto, I. Iwaski,H. Tsuchida, \\ Y. Taguchi, S. Ishiguro, K. Kumagai, T. Yanaka, and M. Mochizuki \\ Research and Development, Advanced Technology Development Center, Kyoritsu \\ Seiyaku Corp., Takamihara, Tsukuba, Ibaraki 300-1252, Japan; Yamagata Prefecture \\ Federation Agricultural Mutual Aid Association, Kitagawara, Nanaura, Yamagata 990- \\ 2171, Japan; Central Veterinary Clinical Center Tokachi Agricultural Mutual Aid \\ Association, Kisen Motomatsu-cho, Obihiro, Hokkaido 080-2231, Japan; K. Kumagai, \\ Sakuraga-oka, Aoba-ku, Sendai, Miyagi 981-0961, Japan; Laboratory of Clinical \\ Microbiology, Advanced Technology Development Center, Kyoritsu Seiyaku Corp., \\ Kudan-minami, Chiyoda-ku, Tokyo 102-0073, Japan
}

Mastitis is the most costly disease of dairy cows throughout the world. Intramammary infections during non-lactating periods can cause mastitis after calving, and the reduction of bacterial counts in the mammary gland is the ideal preventive method. Since lactoferrin has bactericidal effects and priming effects on innate immunity, we investigated whether an intramammary infusion of bovine lactoferrin (bLf) could safely prevent mastitis. A total of 398 healthy mammary quarters of 104 cows were used for the study. Thirty-eight mammary quarters showing high bacterial count in milk were excluded. To evaluate the preventive effect of bLf on clinical mastitis development, the remaining 360 mammary quarters were examined until 3 months after calving. A bLf group was infused once intramammarily with bLf 7-14 d after cessation of milking and the other group was kept as a non-treated control. The numbers of quarters in the bLf group and the control group were 179 and 181, respectively.
During the test period, 4 quarters $(2.2 \%)$ were recognized as having clinical mastitis in the bLf group, in contrast with 20 quarters $(11.0 \%)$ in the control group. In addition, to evaluate the preventive effect of bLf on subclinical mastitis development, 398 mammary quarters of 104 cows were used. The numbers of quarters in the bLf group and the control group were 200 and 198, respectively. In the bLf group, 2 quarters $(1.0 \%)$ were recognized as having subclinical mastitis $7 \mathrm{~d}$ after calving, compared with 15 quarters $(8.7 \%)$ in the control group. Incidence of both clinical and subclinical types of mastitis in the bLf group was significantly lower than the control group. No adverse reaction occurred after the infusion of bLf throughout the study. These results suggest that the infusion of bLf during non-lactating periods has a preventive effect against mastitis development after calving.

\section{Human lactoferrin in milk of transgenic animals}

\author{
E.R. Sadchikova, I.L. Goldman, A.N. Krasnov, S.G. Kadulin, S.G. Georgieva, \\ T.G. Ermolkevich, Ya. G. Gursky, N.V. Gnuchev, and G.P. Georgiev \\ Institute of Gene Biology, Russian Academy of Sciences, Moscow, Russia
}

Human lactoferrin is a type of protein, which may have multiple applications. Therefore, it is critical that it be produced in large quantities. The ultimate goal is to create a technology for its mass production. The future will tell which of the currently developed technologies will provide the necessary production volume of human lactoferrin and which will prove to be the most cost efficient.

The Institute of Gene Biology of the Russian Academy of 
Sciences is currently working on a program of human lactoferrin production from the milk of transgenic goats. The aim is to ensure high concentration of recombinant lactoferrin in the milk of transgenic animals, which will be identical to that found in natural animals. To achieve this goal, gene constructions of human lactoferrin with cDNA were created under the control of various milk gene promoters and other regulatory elements. Testing of gene constructions required over 100 primary transgenic mice and lines of transgenic mice. Those mice were used to research the level of concentration of human lactoferrin in the milk, transgenic inheritance over generations, as well as some other parameters of this human protein.

As a result, the gene construction has been chosen which met all the requirements. This gene construction consists of a genome copy of human lactoferrin controlled by the goat casein gene promoter, which provided for the economically viable level of lactoferrin production in the milk of transgenic mice. At the same time, owing to the positioning effect, the use of this construction caused wide variation of lactoferrin concentration in the milk of primary transgenic animals: from $1.5 \mathrm{~g} / \mathrm{L}$ to $40 \mathrm{~g} / \mathrm{L}$. Two generations of transgenic mice with a high level of lactoferrin production showed concentrations over $20 \mathrm{~g} / \mathrm{L}$. Lactoferrin concentration in the milk of transgenic mice during lactation period varied greatly. Most animals had the highest level of lactoferrin concentration in the middle of their lactation period. Initial research of recombinant lactoferrin produced in the milk of transgenic animals confirmed its quality identity to the human lactoferrin. Natural polymorphism of the lactoferrin protein provoked a secondary question concerning the functional identity of its isoforms. This year, there is task to initiate research that would result in creation of lactoferrin transgenic goats.

\title{
Effect of dietary bovine lactoferrin on tolerance to air exposure stress and response in branchial heat-shock protein under low-salinity conditions in grouper Epinephelus coioides
}

\author{
Yokoyama Saichiro, Koshio Shunsuke, Teshima Shin-ichi, Ishikawa Manabu, \\ Reyes-Catacutan Mae, Takakura Natsuko, and Oshida Kyoichi \\ Laboratory of Aquatic Animal Nutrition, Faculty of Fisheries, Kagoshima University, 4-50- \\ 20 Shimoarata, Kagoshima 890-0056, Japan
}

Augmentation of mass mortality caused by disease and stress has been considered as a serious problem in aquaculture worldwide. Therefore, addition of tolerance against stress for fish is an important task in enabling sustainable aquaculture. Recently, we clarified that dietary bovine lactoferrin (bLF) enhances tolerance to high-temperature stress in Japanese flounder, Paralichthys olivaceus; however, there are no reports on the effect in other important species. In present study, dietary bLF was administrated to juvenile grouper (initial mass $=3.0 \mathrm{~g}$ ) to investigate its effects on growth response, mucus presence, tolerance to air exposure, and response in branchial heat-shock protein under low-salinity conditions. Test diets containing 4 different concentrations of bLF $(0,400,800$, and $1200 \mathrm{mg} \mathrm{bLF} / \mathrm{kg}$ diet $)$ were fed to specimens for $30 \mathrm{~d}$. After feeding trial, amounts of secreted mucus on the body surface were measured while fish were exposed to the air for $60 \mathrm{~min}$ to obtain the recov- ery from respiratory distress condition. Furthermore, fish were exposed to low-salinity $(3.5 \mathrm{ppt})$ seawater for 0 (nostress) and $6 \mathrm{~h}$. After low-salinity seawater exposure, the levels of heat-shock protein 70 family proteins (HSP70s) in the branchial lamella were measured by enzyme-linked immunosorbent assay. Dietary bLF did not affect growth rate of this species. Mucus secretion significantly increased in all bLF-supplemented groups compared with that in the $0 \mathrm{mg}$ bLF group. Recovery rate from the air-exposure stress test increased with increasing dietary bLF level. Under the low-salinity exposure, significantly higher levels of HSP70s were detected in 800 and $1200 \mathrm{mg}$ bLF-supplemented groups than those in the bLF $0 \mathrm{mg}$ group after both 0 and $6 \mathrm{~h}$ exposure. These results suggest that dietary bLF enhances tolerance to physiological stress, and is an effective means for evaluating the quality of juvenile grouper. 


\title{
Therapeutic effects of bovine lactoferrin on staphylococcal mastitis during non-lactating period
}

\author{
K. Kai, Y. Komine, K. Komine, M. Ohta, and K. Kumagai \\ Research and Development, Advanced Technology Development Center, Kyoritsu \\ Seiyaku Corporation, Takamihara, Tsukuba, Ibaraki 300-1252, Japan; Intelligent Cosmos \\ Research Institute, Minamiyoshinari, Aoba-ku, Sendai, Miyagi 989-3204, Japan; M. Ohta, \\ Choumeigaoka, Izumi-ku, Sendai, Miyagi 981-3212, Japan; K. Kumagai, Sakuraga-oka, \\ Aoba-ku, Sendai, Miyagi 981-0961, Japan
}

During the non-lactating period, a few months before calving, there are increases in the concentration of bovine lactoferrin (bLf), complement components, and phagocytes in mammary gland secretions. These may play an important role in host defense against bacteria. We investigated whether an intramammary infusion of bLf could effect the cure rate of mastitis and mammary gland immunity. To evaluate the clinical effects of bLf on staphylococcal mastitis in Holstein cows during the early non-lactating period, 41 mammary quarters were selected randomly from 36 cows on 3 dairy farms. Twelve quarters were infused intramammarily with bovine lactoferrin. Twenty-nine quarters were infused with an antibiotic as a control. In the bLf group, $91.7 \%$ of mastitic quarters were cured $7 \mathrm{~d}$ after calving, compared with $48.3 \%$ in the control group. Furthermore, the changes in mammary secretion induced by the infusion of bLf were investigated. Mean numbers of staphylococci in mammary gland secretions were significantly decreased in 5 bLf-infused quarters and in 5 antibiotic-infused control quarters. Unlike in the control quarters, the mean total cell concentration in the mammary gland secretions increased in bLf-infused quarters. Similar results were obtained in 6 healthy quarters that were infused with bLf. In these quarters, the cell population contained mainly phagocytes such as polymorphonuclear leukocytes and cells positive for CD11b, which is known as a complement receptor. The mean concentration of C3 in mammary gland secretions was significantly increased in 5 mastitic quarters infused with bLf, but showed no significant change in 5 mastitic control quarters. These results suggest that bLf treatment for staphylococcal mastitis in the early non-lactating period might increase the rate of cure through the induction of innate immunity in the host.

\section{NMR structural studies of bovine and human lactoferrampin, novel antimicrobial peptides from lactoferrin}

\author{
E.F. Haney, F. Lau, and H.J. Vogel \\ Department of Biological Sciences, University of Calgary, 2500 University Dr. NW, \\ Calgary, AB T2N 4N1, Canada
}

Lactoferrin has been identified as a protein with numerous functions, one of which is the ability to inhibit bacterial growth. A pepsin digestion fragment, known as lactoferricin, has already been implicated in the antimicrobial activity of lactoferrin. It is found in the highly cationic N-terminal segment of lactoferrin that distinguishes this protein from other transferrins. Recently, a novel antimicrobial peptide, lactoferrampin, was identified in the sequence of bovine lactoferrin. This fragment is a cationic stretch of 17 amino acids that lies adjacent to lactoferricin on the surface of the N-terminal lobe of the lactoferrin protein. The bovine lactoferrampin peptide (WKLLSKAQEKFGKNKSR) corresponds to residues 268-284 of bovine lactoferrin. The related human peptide (WNLLRQAQEKFGKDKSP) corresponds to residues $269-285$ of human lactoferrin and is $70 \%$ homologous to the bovine form, but is less cationic. These 2 peptides have been synthesized and their solution structures have been determined in SDS and DPC micelles using 2-dimen- 
sional nuclear magnetic resonance (NMR) techniques. The human and bovine peptides were relatively unstructured in water; however, both formed amphipathic helices in SDS micelles. The amphipathic helix is a known motif in many other antimicrobial peptides where the positively charged residues interact with the negatively charged bacterial surface and the hydrophobic residues are capable of interacting with the hydrophobic tails of the peptide-based antibiotics that are highly selective and efficient against pathogenic bacteria.

\title{
Long-term follow-up of chronic hepatitis C patients administered oral lactoferrin
}

\author{
K. Higami, K. Ishii, M.L. Shinohara, T. Ikehara, H. Nagai, M. Watanabe, Y. Sumino, \\ K. Miki, and K. Yamauchi \\ Division of Gastroenterology and Hepatology, Toho University School of Medicine, Tokyo; \\ Nutritional Science Laboratory, Morinaga Milk Industry Ltd, Kanagawa, Japan
}

We have already reported that oral administration of bovine lactoferrin (bLF) to chronic hepatitis $\mathrm{C}(\mathrm{CHC})$ patients produced a Th1-cytokine dominant environment in the peripheral blood for up to 3 months. The present study attempted to clarify the effects of long-term administration of bLF on serum parameters and a developing rate of hepatocellular carcinoma (HCC) in CHC patients. Daily, $600 \mathrm{mg}$ of bLf (Morinaga Milk Industry Ltd.) was orally administered to $25 \mathrm{CHC}$ patients (bLf group) and they were followed for a median of 56 months (range: 25-72 months). Serum parameters and a developing rate of HCC were compared with those of a control group, which included sex- and age-matched $\mathrm{CHC}$ patients (a median of 55 months; range: 12-72 months). HCC was histopathologically diagnosed us- ing tumor biopsy specimens. Serum parameters included ALT, iron, ferritin, and quantitative HCV-RNA by RT-PCR (Amplicor v. 2.0; Roche, Basel, Switzerland).

Serum parameters were not significantly changed during the period, nor were those of the control. HCCs were developed in 3 patients from the bLF group $(2.7 \%$ per person per year) and in 3 patients from the control group $(2.9 \%$ per person per year). The developing rate of HCC was not different between 2 groups.

These results suggest that oral bLF may not prevent developing $\mathrm{HCC}$, although further follow up for those patients and investigation will be needed.

\section{Effects of lactoferrin on adipogenesis in 3t3-I1 cells and obesity mice}

\author{
M.S. Nam, M.C. Cho, H.C. Bae, and D.Y. Yoon \\ Laboratory of Milk Food Biochemistry, Division of Animal Science and Resources, \\ College of Agriculture and Life Sciences, Chungnam National University, Daejeon 305- \\ 764, Korea; Laboratory of Cell Biology, Korea Research Institute of Bioscience and \\ Biotechnology, PO Box 115, Daejeon 305-333, Korea
}

This experiment was carried out to investigate effects of the multifunctional iron-binding glycoprotein lactoferrin on adipogenesis and obesity mice. Lactoferrin inhibited lipid drop formation in differentiated 3T3-L1 adipocytes induced by insulin, dexamethasone, and isobutylmethylxanthin. RTPCR revealed that the lactoferrin receptor was expressed in 3T3-L1 adipocytes. Oral administration into BKS.cg- $m^{+/+}$ Lepr $^{\mathrm{db}}$ obesity mice showed that there was no effect on body mass increase at high doses of $\mathrm{Lf}(10 \mathrm{mg} / \mathrm{d})$, whereas body mass was increased by as much as $30 \%$ in control mice (not administrated) and those administered a low dose of Lf $(5 \mathrm{mg} / \mathrm{d})$. In addition, cholesterol level was reduced in mice treated with a high dose of $\mathrm{Lf}(10 \mathrm{mg} / \mathrm{d})$. The results suggest that Lf plays a potentially important role in reducing body mass and regulating fat metabolism. 


\title{
Lactoferrin in dentistry
}

\author{
Tamaki Yukimichi and Ando Kunio \\ Showa University School of Dentistry, Department of Oral Biomaterials and Technology, \\ 1-5-8 Hatanodai, Shinagawa-ku, Tokyo 142-8555, Japan
}

Although a large amount of research has been carried out on the structure and function of lactoferrin since its discovery in 1939, there are a few studies relating to its dental application. This study deals with its clinical efficacy on such oral diseases as periodontitis, xerostomia, halitosis, stomatitis, angulitis, and glossodynia, since lactoferrin has immuno-potentiating properties and shows anti-inflammatory and antinociceptive activities in animal studies.

Bovine milk lactoferrin used in this study was the product of Murray Goulburn Co. (Brunswick, Australia). The enteric-coated capsules and plain-flat oral patches $(8 \mathrm{~mm}$ diameter, $1 \mathrm{~mm}$ thick) were supplied from NRL Pharma Inc. (Kawasaki, Japan). The lactoferrin contentof each was $100 \mathrm{mg} / \mathrm{capsule}$ and $30 \mathrm{mg} / \mathrm{patch}$, respectively. The patients enrolled in this study signed their informed consent after an oral explanation of the experiment. Fresh breath was collected from patients with halitosis early in the morning and volatile sulfur compounds in breath were determined by gas chromatography.

The capsules were used for the treatment of severe periodontitis, zerostomia, and halitosis and the patches were used for treatment of stomatitis, angulitis, and glossodynia. Treatment with these regimens was highly efficacious without any side effects. Especially, the patch directly applied on injured regions reduced pain within 5 min and seemed to stimulate wound healing. The treatment with the capsules was also efficacious for severe periodontitis, xerostomia, and halitosis; the treatment with 3-6 capsules/d rapidly improved dry-mouth caused either by Sjogren syndrome or induced by drugs. Moreover, treatment resulted in significant reduction of volatile sulfur compounds within a few days. Consequently, lactoferrin is highly efficacious both when applied topically on injured oral mucosa and when taken orally as enteric-coated capsules for treating periodontitis, xerostomia, and halitosis.

\section{Killing of pneumococci by apolactoferrin is dependent on cleavage of apolactoferrin by the pneumococcal surface serine protease PrtA}

\author{
Shaper Mirza, William H. Benjamin Jr., and David E. Briles \\ Departments of Microbiology and Pathology, University of Alabama, Birmingham, $A L$, \\ USA
}

Apolactoferrin (ALF) is known to kill pneumococci and this killing is largely, but not completely, blocked by pneumococcal surface protein A (PspA). We now report more recent studies showing that the killing of pneumococci by ALF could be blocked by serine protease inhibitors such as pneylmethylsulfonylfloride (PMSF) and soyabean trypsin inhibitor (SBT). Analysis of the sequenced TIGR4 genome demonstrated the presence of 3 putative cell-surface-associated proteases including PrtA. PrtA is a member of the subtilisin-like family of serine proteases (Beth, G., et al. 2001. FEMS Microbiol Lett. 205(1): 99-104), which is released from the bacterial surface as an active enzyme. We constructed a prtA knockout mutant of strain D39 and found that it almost completely blocked killing by apolactoferrin. Further study showed that when apolactoferrin is added to wild-type and PrtA mutant D39, the ALF in solution with the wild-type D39 is cleaved, whereas the apolactoferrin in solution with the PrtA mutant pneumococci was not cleaved. It is likely that the cleavage by PrtA resulted in the generation of low-molecular-weight lactoferricins that were the actual cause of bacterial death. Finally, when we examined the apolactoferrin bound to PspA, we found that it was not cleaved by either strain. These findings indicate that binding to PspA blocks cleavage of ALF by PrtA. Thus, PspA's ability to inhibit killing of pneumococci by ALF could easily be because it prevents cleavage of ALF by PrtA. 


\title{
Cleavage of human lactoferrin by a surface- associated serine protease (PrtA) of Streptococcus pneumoniae
}

\author{
Shaper Mirza and David E. Briles \\ Department of Microbiology University of Alabama, Birmingham, AL, USA
}

Serine protease activity is critical for apolactoferrin (ALF) to be able to kill pneumococci. Phenylmethyl-sulfonylfloride (PMSF), a serine protease inhibitor, at a concentration of $7.5 \mathrm{mmol} / \mathrm{L}$ is able to block killing by $3.1 \mu \mathrm{mol} / \mathrm{L}$ apolactoferrin. PMSF was not, however, able to block killing by lactoferricin, the N-terminal bactericidal fragment of lactoferrin. These observations suggested that killing of pneumococci by apolactoferrin may depend on the ability of a bacterial protease to cleave a lactoferricin peptide from ALF that actually mediates the killing. Evidence that growing pneumococci could degrade lactoferrin came from our observation that incubation of $50 \mu \mathrm{g} / \mathrm{mL}$ apolactoferrin from human milk with growing pneumococci resulted in a reduction in molecular mass of the apolactoferrin by about $10 \mathrm{kDa}$. A targeted mutation resulting in the disruption of the prtA gene inhibited the degradation of apolactoferrin. We also observed that capsular type 2 and 19F strains with prtA mutations became relatively resistant to killing by apolactoferrin ( $p$ value $>0.001$ ). Our interpretation of these data are that prtA acts to cleave lactoferricin from apolactoferrin and the released lactoferricin is probably the mechanism by which ALF kills bacteria.

\section{PspA inhibits the antibacterial effect of lactoferrin on Streptococcus pneumoniae}

\author{
David E. Briles and Shaper Mirza \\ Department of Microbiology, University of Alabama, Birmingham, AL, USA
}

The only surface protein on pneumococci that binds significantly to lactoferrin is pneumococcal surface protein A (PspA). Apolactoferrin binds to the N-terminal half of PspA. PspA binds to both apo- and holo-lactoferrin. This binding, however, is not a mechanism for iron acquisition, since hololactoferrin cannot serve as an iron source for pneumococci, which acquire their iron from heme. Apolactoferrin, but not hololactoferrin, is able to kill pneumococci during a 1 to $2 \mathrm{~h}$ incubation in vitro. PspA very effectively inhibits the killing of pneumococci by apolactoferrin and by lactoferricin (an N-terminal fragment of lactoferrin). We have demonstrated this by comparing the susceptibility to apolactoferrin and lactoferricin of wild-type pneumococci with mutants of pneumococci lacking PspA expression. We have also shown that the soluble N-terminal half of PspA can inhibit killing of pneumococci by apolactoferrin. Anti- body to PspA is able to enhance killing of pneumococci by apolactoferrin. We have shown that antibody to PspA inhibits binding of apolactoferrin to PspA, thus explaining how the antibody may enhance killing by apolactoferrin. Since lactoferricin is more bactericidal on a molar basis than apolactoferrin, we feel that the mechanism by which apolactoferrin kills pneumococci is by first being cleaved into lactoferricin. We have shown that for pneumococci to be killed by apolactoferrin hey must possess the surface serine protease PrtA. We have also shown that this enzyme can degrade apolactoferrin. Thus, it would appear that a bacterial protease is required for the susceptibility of bacteria to killing by apolactoferrin. The ability of apolactoferrin to kill pneumococci may explain in part why pneumococci invade the submucosa during nasal colonization. 


\title{
Fusion expression of bovine lactoferricin in Escherichia Coli
}

\author{
Jianhua Wang, X. Feng, A. Shan, D. Teng, Y. Yao, Y. Shao, S. Liu, and F. Zhang \\ Gene Engineering Laboratory, Feed Research Institute, Chinese Academy of Agricultural \\ Sciences, Beijing 100081, China; Animal Nutrition Research Institute, Northeast \\ Agricultural University, Harbin 150030, China; Department of Pharmaceutical Science, \\ University of Toronto, Toronto, ON M5S 2S2, Canada; College of Food Science and \\ Technology, Huazhong Agricultural University, Wuhan 430070, China; College of Food \\ Science and Technology, Nanjing Agricultural University, Nanjing 210095, China
}

The drug-resistance problem has been growing with use of current antibiotics in the feed and medical industries. LfcinB, a 25 amino acid antibacterial peptide derived from bovine lactoferrin, is a potential alternative to antibiotics. According to the bias of codon use in Escherichia coli, the gene encoding LfcinB has been chemically synthesized and ligated into the expression vector pGEX-4T-2. The antibacterial peptide was fused with GST with a protease cleavage site in between them. Two constructs with different cleavage sites were made. One construct, GEX-Th-LCB, contained a thrombin cleavage site provided by the vector. In the second construct, GEX-Th-Xa-LfcinB, a factor Xa cleavage site was introduced after the thrombin cleavage site. The GSTTh-LCB protein was efficiently cleaved by thrombin and the recombinant LfcinB showed antibacterial activity. However, the fusion protein containing the factor Xa recognition site could not be cleaved by factor $\mathrm{Xa}$ in this study. The high level of expression of LfcinB in E. coli provides a possible method for producing LfcinB in large amounts. This project is funded by Chinese National Hi-Tech Research and Development Program Nos. 2004AA246040 and 2003AA241160-01.

\section{The efficacy of bovine lactoferrin and ifn- $\alpha \mathbf{2 b}$ combination therapy following 24-week regimen of ribavirin plus ifn- $\alpha \mathbf{2 b}$ for chronic hepatits $C$ patients infected with genotype $1 \mathrm{~b}$ and high viral load}

\author{
K. Ishii, Y. Sumino, M. Kogame, K. Higami, M.I. Shinohara, K. Matsumaru, \\ M.A. Shinohara, H. Nagai, M. Watanabe, K. Miki, K. Yamauchi, and S. Teraguchi \\ Division of Gastroenterology and Hepatology, Toho University School of Medicine, Tokyo, \\ Japan; Nutritional Science Laboratory, Morinaga Milk Industry Co, Ltd, Kanagawa, Japan
}

The aim of this study was to clarify the efficacy of a regimen of oral bovine lactoferrin (bLF) and interferon- $\alpha 2 b$ (IFNa-2b) combination therapy following a 24 week course of ribavirin plus IFN- $\alpha 2$ b for chronic hepatitis $\mathrm{C}(\mathrm{CHC})$ patients. Forty patients with biopsy-proven $\mathrm{CHC}$ who were infected with genotype $1 \mathrm{~b}$ and had a high viral load were eligible for the study. Their baseline HCV-RNA quantified by RT-PCR (Amplicor v. 2.0; Roche, Basel, Switzerland) in serum was more than $101 \mathrm{KIU} / \mathrm{mL}$. A 24 week regimen of IFNa-2b (6 MU daily for 2 weeks, followed by 6 MU 3 times/week for 22 weeks) and ribavirin (daily dose of 600-
$800 \mathrm{mg}$ according to body mass) was given to all patients. After week 24, 13 patients (a median baseline HCV-RNA of $385 \mathrm{KIU} / \mathrm{mL}$ ) were discontinued (control group), 13 patients (a median baseline HCV-RNA of $430 \mathrm{KIU} / \mathrm{mL}$ ) were given $600 \mathrm{mg} /$ day of oral bLF (Lactoferrin active ${ }^{\mathrm{R}}$, Morinaga) and 3-6 MU IFNa-2b for a median period of 6 months (bLf group), and the remaining 14 patients (a median baseline HCV-RNA of $490 \mathrm{KIU} / \mathrm{mL}$ ) were given 3-6 MU IFNa$2 \mathrm{~b}$ for a median period of 6 months (IFN group).

The negativity of serum HCV-RNA using a qualitative method (Amplicor) at weeks 4, 12, and 24 was seen in 1, 3, 
and 4 patients from the control group; in 1, 3, and 6 patients from the bLf group; and in 2, 6, and 8 patients from the IFN group, respectively. Sustained viral response (serum HCVRNA at 24 weeks after the end of therapy was negative) was seen in 1 patient from the control group, in 4 patients from the bLf group, and in 2 patients from the IFN group.
These results indicate that bLF and IFNa-2b combination therapy following a 24-week course of ribavirin plus IFNa$2 b$ is effective for $\mathrm{CHC}$ patients infected with genotype $1 \mathrm{~b}$ and a high viral load. 
Copyright of Biochemistry \& Cell Biology is the property of NRC Research Press and its content may not be copied or emailed to multiple sites or posted to a listserv without the copyright holder's express written permission. However, users may print, download, or email articles for individual use. 